\title{
Regulation of HIV-I and IL-2 transcription by inducible CAMP early repressor (ICER) Josef Bodor*
}

Address: Columbia University, College of Physicians and Surgeons, Department of Medicine, New York, New York, 10032, USA

* Corresponding author

from 2006 International Meeting of The Institute of Human Virology

Baltimore, USA. 17-2I November, 2006

Published: 21 December 2006

Retrovirology 2006, 3(SuppI I):S8I doi:I0.II86/I742-4690-3-SI-S8I

(C) 2006 Bodor; licensee BioMed Central Ltd.

HIV-1 infection of human monocytes induces production of prostaglandin E2 (PGE2) in vitro and in vivo, which has capacity to make the bystander $\mathrm{T}$ cells unresponsive (anergic). ICER, a potent transcriptional repressor induced by PGE2 leads to transcriptional attenuation of interleukin-2 (IL-2) and an induction of T cell suppression. We propose that this HIV-1 triggered mechanism may subvert physiologically relevant suppression of IL-2 by regulatory CD4+CD25+T (Treg) cells. Our preliminary studies suggest that the suppressive mechanism conveyed by Treg cells stems from coexpression of ICER and Foxp3, which inhibits IL-2 expression. Moreover, naïve CD4+CD25- responder $\mathrm{T}$ cells retrovirally transduced with Foxp3 can induce the accumulation of ICER and replace natural Treg cells. Importantly, ICER expression is induced in activated responder T cells early and correlates with a sharp decrease of IL-2 expression. Our preliminary studies indicate that ICER inhibits HIV-1 LTR expression and binds the cyclic responsive element (CRE)-like motifs termed DSE positioned downstream of transcription initiation site of HIV-1 LTR. Moreover, in monocytes PGE2 induced transcriptional attenuation of CCR5 expression tightly correlates with upregulation of ICER. We conclude that ICER is a critical component of Treg-mediated inhibitory function that affects transcriptional attenuation of IL-2 production in T cells and that HIV-1 subverts this mechanism via PGE2 and/or Tat-mediated induction of ICER. 